\title{
Actuator fault diagnosis of singular delayed LPV systems with inexact measured parameters via PI unknown input observer
}

\author{
Amir Hossein Hassanabadi ${ }^{1, *}$, Masoud Shafiee ${ }^{1}$ and Vicenc Puig ${ }^{2}$ \\ ${ }^{1}$ Department of Electrical Engineering, Amirkabir University of Technology (Tehran Polytechnic), 424, Hafez Ave., Tehran, Iran \\ ${ }^{2}$ Automatic Control Department, Technical University of Catalonia (UPC), Pau Gargallo, 5, 08028 Barcelona, Spain \\ *a.hassanabadi@aut.ac.ir
}

\begin{abstract}
In this paper, actuator fault diagnosis of singular delayed linear parameter varying (SDLPV) systems is considered. The considered system has a time varying state delay and its matrices are dependent on some parameters that are measurable online. It is assumed that the measured parameters are inexact due to the existence of noise in real situations. The system with inexact measured parameters is converted to an uncertain system. Actuator fault diagnosis is carried out based on fault size estimation. For this purpose, the system is transformed to a polytopic representation and then a polytopic proportional integral unknown input observer (PI-UIO) is designed. The proposed observer provides simultaneous state and actuator fault estimation while attenuating, in the $H_{\infty}$ sense, the effects of input disturbance, output noise and the uncertainty caused by inexact measured parameters. The design procedure of PI-UIO is formulated as a convex optimization problem with a set of LMI constraints in the vertices of the parameter domain, guaranteeing robust exponential convergence of the PI-UIO. The efficiency of the proposed method is illustrated with an electrical circuit example modeled as a SDLPV system.
\end{abstract}

\section{Introduction}

Modern systems are becoming more complex and consequently the need for safety, reliability and higher performance has motivated the research on fault diagnosis systems. Early and correct detection, isolation and estimation of the sizes of the faults are needed to perform suitable fault tolerant actions in order to prevent highly sophisticated systems from being stopped, damaged or causing hazards to human operators. Fault diagnosis methods are categorized into model-based and model-free methods. In model-based fault diagnosis, the system operation is compared with what is expected from its non-faulty model exploiting the existing analytic redundancy. Observer-based methods are very common in the category of model-based methods which have attracted much attention in the recent years. Several kinds of observers have been applied in the field of fault diagnosis. Unknown Input Observers (UIOs)[1-3] can provide faulty situation indicators called residuals decoupled from unknown inputs (disturbances) to carry out fault detection. Fault isolation can be carried out based on a suitable bank of UIOs, each decoupled from a subset of faults. The use of these observers for fault estimation has just recently been proposed $[4,5]$. Fault estimation (reconstruction) is important for adjusting fault tolerant actions in the system also providing a straightforward approach for fault diagnosis that could avoid the fault detection and isolation steps. The Proportional Integral (PI) observer has been used to estimate the sizes of the faults. This observer has been used for sensor fault estimation in [6] and for actuator fault estimation in [7]. Actuator and sensor faults have been simultaneously 
reconstructed by the PI observer in [8] with the aid of the descriptor approach. The authors in [9] have modified this observer to include multiple integral actions in order to estimate the fault derivatives but the proportional multiple integral (PMI) observer suffers from higher noise sensitivity.

Singular systems (which are also called descriptor systems, generalized systems, differential-algebraic systems and semi-state systems in the literature) arise from a natural approach in system modeling when as e.g. static mass/energy balances are involved [10]. They are successfully used to model various electrical, mechanical, economical, chemical and biological systems (see $[10,11]$ and references therein). Actuator fault diagnosis of linear and nonlinear singular systems have been performed in [12] and [13], respectively. Linear parameter varying (LPV) systems which can provide a good approximation of nonlinear systems have attracted the attention of the researchers. LPV representation which has been originated from the gain-scheduling control systems, allows the use of linear systems methodologies in nonlinear systems. In particular, singular LPV systems, which can model nonlinear systems with algebraic constraints, have been used in modelling different processes [14, 15]. Actuator fault reconstruction has been developed for LPV systems based on learning observers in [16]. Constant and time varying actuator fault diagnosis of singular LPV systems have been considered in [7] and [14], respectively, considering both PI and adaptive observers with the assumption that the exact knowledge of the parameters is available for observer scheduling. Fault diagnosis of singular LPV systems in the case of an unmeasurable set of parameters is considered in [15] with the descriptor system approach to estimate sensor faults and in [17] with the $H_{-} / H_{\infty}$ approach to simultaneously guarantee the fault sensitivity and robustness requirements of the fault diagnosis observer.

Delay appears in the dynamics of many real processes (as e.g., when considering transport phenomena or communications networks) being the cause for instability and performance degradation. Fault diagnosis of delayed systems is an active research area. Fault estimation is a direct method for fault diagnosis that can be addressed with several methods Fault estimation in delayed systems has been carried out with the sliding mode observer (SMO) [18], descriptor system approach [19], adaptive observer [20] and PI observer [21]. Singular delayed linear parameter varying (SDLPV) systems have been recently considered. Robust stability and filtering of continuous-time SDLPV systems are considered in [22, 23] while the stability and stabilization criteria for discrete-time counterparts are considered in [24]. In [25], the authors have designed UIO for these systems in the case of exact measured parameters and have used the proposed observer for actuator fault detection and isolation purposes. The problem of fault diagnosis in SDLPV systems with inexact measured parameters has not been considered yet to the best of the authors' knowledge.

The goal of this paper is to design a polytopic PI unknown input observer (PI-UIO) to address the problem of actuator fault estimation for SDLPV systems in the case of inexact measured parameters. Because the measured parameters that are used for the observer scheduling are inexact, some uncertainty is induced in the estimation process. The inexactness of the parameter measures is taken into account with representing the system by an uncertain system formulation. The designed observer can also be used for simultaneous state and actuator fault estimation. The fault estimation error dynamics is obtained with the assumption that the actuator faults are constant or slow varying and then it is augmented with state estimation error dynamics. The stability of this augmented system and the attenuation of the effects of disturbance, noise and the uncertainty caused by inexact parameters is addressed with a related Bounded Real Lemma (BRL) for SDLPV systems. The design procedure of PI-UIO is formulated as a convex optimization problem with a set of LMI constraints in the vertices of the parameter domain polytope. This paper is the extension of the results of [7] to the case which delayed dynamics is present in the singular LPV system. Another important contribution of this paper is that it considers inexact measured parameters which is more realistic 
than in $[7,25]$ where the case of exact parameter measures is assumed. The preliminary results of this paper (PI-UIO design for SDLPV systems with exact measured parameters) are presented in [26].

This paper is structured as follows: In Section 2, the problem under consideration is formulated. A suitable PI-UIO for SDLPV systems is proposed in Section 3. In Section 4, the design procedure of PI-UIO and the fault diagnosis with this observer is presented. In Section 5, the effectiveness of the proposed method will be illustrated and discussed with an electrical circuit example and Section 6 draws the paper conclusions.

Notation: The notation used in this paper is standard. $R$ is the set of real numbers. $I_{n}$ is the $n$-dimensional identity matrix. For a matrix $X, X^{T}$ indicates its transpose. $X^{-1}$ is the inverse and $X^{+}$is the pseudo inverse of $X$.* is used to show the elements induced by symmetry in a symmetric matrix. $\operatorname{sym}\{A\}$ is a short notation for $A+A^{T}$. For a symmetric matrix $X, X>0(X<0)$ shows that it is positive (negative) definite. For a square integrable function $x(t)$, its $L_{2}$-norm is defined as $\|x(t)\|_{2}=\sqrt{\int_{0}^{\infty} x(t)^{T} x(t) d t}$.

\section{Problem formulation}

In this paper, a SDLPV system with the following formulation is considered:

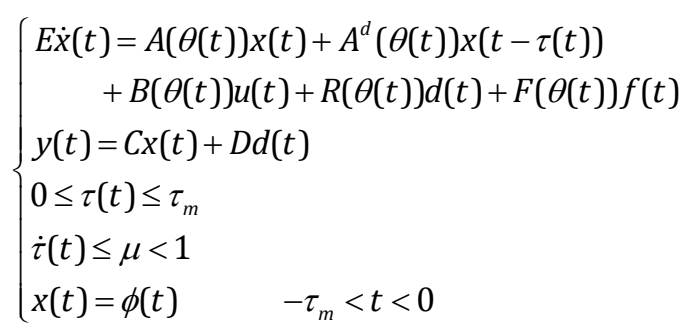

where $\quad x(t) \in R^{n}, \quad u(t) \in R^{k_{u}}, \quad y(t) \in R^{m}, \quad d(t) \in R^{k_{d}}$ and $f(t) \in R^{k_{f}}$ are state vector, input vector, output vector, disturbance vector and actuator fault vector, respectively. In (1), $E \in R^{n \times n}$ is a constant square matrix that may have rank deficiency $(\operatorname{rank}(E)=r \leq n) . \quad A(\theta(t)), A^{d}(\theta(t)), B(\theta(t))$, $R(\theta(t))$ and $F(\theta(t))$ are matrices with appropriate dimensions which depend affinely on the time varying parameter vector $\theta(t) \in R^{l}$ that is real time measurable. $C$ and $D$ are two constant matrices with appropriate dimensions. $\tau(t)$ is a time varying state delay. $\tau_{m}$ and $\mu$ are the maximum values of the delay and its rate of change. $\phi(t)$ is a continuous vectorvalued initial function.

Assumption 1. The time varying parameter vector belongs to the hyperbox $\Omega$ defined as follows:

$$
\Omega=\left\{\theta(t) \mid \theta_{k}^{m} \leq \theta_{k}(t) \leq \theta_{k}^{M} \text { for } k=1, \cdots, l\right\}
$$

in which $\theta_{k}^{m}$ and $\theta_{k}^{M}$ represents the minimum and maximum values of each parameter.

Definition 1 [10]. The matrix pencil $(E, A)$ is regular if $\operatorname{det}(s E-A)$ is not identically zero.

Definition 2 [10]. The matrix pencil $(E, A)$ is impulse-free if $\operatorname{deg}(\operatorname{det}(s E-A))=\operatorname{rank}(E)$.

Definition 3 [23]. System (1) is regular and impulse-free if the matrix pencils $(E, A(\theta(t)))$ and $\left(E, A(\theta(t))+A^{d}(\theta(t))\right)$ are regular and impulse-free for all the values of $\theta(t)$ in the domain defined in (2).

Definition 4 [23]. System (1) is admissible if it is regular, impulse free and stable.

Assumption 2. System (1) is assumed to be admissible.

Assumption 3. In system (1), $\operatorname{rank}\left[\begin{array}{l}E \\ C\end{array}\right]=n$.

Assumption 4. It is assumed that the faults are constant or slow varying $(\dot{f}(t) \cong 0)$. 
System (1) can be formulated in the following polytopic form:

$$
\left\{\begin{aligned}
E \dot{x}(t) & =\sum_{i=1}^{h} \rho_{i}(\theta(t))\left[A_{i} x(t)+A_{i}^{d} x(t-\tau(t))\right. \\
& \left.+B_{i} u(t)+R_{i} d(t)+F_{i} f(t)\right] \\
y(t) & =C x(t)+D d(t)
\end{aligned}\right.
$$

where $h=2^{l}$ is the number of subsystems in the polytopic representation. $A_{i}, A_{i}^{d}, B_{i}, \quad R_{i}$ and $F_{i}$ for $i=1, \cdots, h$ are matrices describing the dynamics of the subsystem in the $i^{\text {th }}$ vertex of the hyperbox. $\rho_{i}(\theta(t))$ for $i=1, \cdots, h$ are different subsystem weights which satisfy the following convex property:

$$
\begin{aligned}
& 0 \leq \rho_{i}(\theta(t)) \leq 1 \\
& \sum_{i=1}^{h} \rho_{i}(\theta(t))=1 .
\end{aligned}
$$

Remark 1. The polytopic representation matrices $A_{i}, A_{i}^{d}, B_{i}$, $R_{i}$ and $F_{i}$ and the subsystem weights $\rho_{i}(\theta(t))$ for $i=1, \cdots, h$ can be computed with the method presented in [27] with any number of parameters in the SDLPV system (1).

\section{PI-UIO formulation and preliminaries}

In order to estimate the states and actuator faults in system (3), the following PI-UIO is proposed:

$$
\left\{\begin{aligned}
\dot{z}(t) & =\sum_{j=1}^{h} \rho_{j}(\hat{\theta}(t))\left[N_{j} z(t)+N_{j}^{d} z(t-\tau(t))\right. \\
& \left.+L_{j} y(t)+L_{j}^{d} y(t-\tau(t))+G_{j} u(t)+W_{j} \hat{f}(t)\right] \\
\hat{x}(t) & =z(t)+H_{2} y(t) \\
\hat{y}(t) & =C \hat{x}(t) \\
\dot{\hat{f}}(t) & =\sum_{j=1}^{h} \rho_{j}(\hat{\theta}(t)) \Lambda_{j}(y(t)-\hat{y}(t)) \\
z(t) & =0 \quad-\tau_{m}<t<0
\end{aligned}\right.
$$

where $\hat{x}(t) \in R^{n}, \quad \hat{y}(t) \in R^{m}, \quad z(t) \in R^{n}$ and $\hat{f}(t) \in R^{k_{f}}$ are state estimate, output estimate, observer state and fault estimate, respectively. $N_{j}, N_{j}^{d}, L_{j}, L_{j}^{d}, G_{j}, W_{j}, \Lambda_{j}$ and $H_{2}$ are observer matrices with appropriate dimensions such that the procedure for calculating them will be presented in the following. In this paper, it is assumed that the measured values of the parameters $(\hat{\theta}(t))$ are different from real values of the parameters $(\theta(t))$ which are unknown. The observer (6) is scheduled with $\rho_{j}(\hat{\theta}(t))$ calculated based on the measured values of the parameters. The difference between the scheduling functions of system (3) and observer (6) imposes an uncertainty in the estimation procedure. Thus, the goal is to design observer (6) robust against these uncertainties in addition to noise and disturbance. In order to consider the uncertainty induced by inexact measured parameters and facilitate the PI-UIO design, the method proposed in [28] is extended. This is done by means of some manipulations of system (3) that allow reformulating it as an uncertain system as follows:

$$
\left\{\begin{aligned}
E \dot{x}(t) & =\sum_{i=1}^{h} \sum_{j=1}^{h} \rho_{i}(\theta(t)) \rho_{j}(\hat{\theta}(t))\left[\tilde{A}_{i j} x(t)\right. \\
& \left.+\tilde{A}_{i j}^{d} x(t-\tau(t))+\tilde{B}_{i j} u(t)+\tilde{F}_{i j} f(t)+R_{i} d(t)\right] \\
y(t)= & C x(t)+D d(t)
\end{aligned}\right.
$$

where the following notation is used:

$$
\begin{array}{cc}
\tilde{A}_{i j}=A_{j}+\Delta A_{i j}, & \Delta A_{i j}=A_{i}-A_{j}, \\
\tilde{A}_{i j}^{d}=A_{j}^{d}+\Delta A_{i j}^{d}, & \Delta A_{i j}^{d}=A_{i}^{d}-A_{j}^{d}, \\
\tilde{B}_{i j}=B_{j}+\Delta B_{i j}, & \Delta B_{i j}=B_{i}-B_{j}, \\
\tilde{F}_{i j}=F_{j}+\Delta F_{i j}, & \Delta F_{i j}=F_{i}-F_{j} .
\end{array}
$$

The state estimation error is:

$$
e(t)=x(t)-\hat{x}(t)
$$

that according to (6) and (7) becomes: 


$$
\begin{gathered}
e(t)=x(t)-z(t)-H_{2} C x(t)-H_{2} D d(t) \\
=\left(I_{n}-H_{2} C\right) x(t)-z(t)-H_{2} D d(t) .
\end{gathered}
$$

If there exists a matrix $H_{1} \in R^{n \times n}$ that satisfies the following condition:

$$
H_{1} E=I_{n}-H_{2} C
$$

then (13) is converted to

$$
e(t)=H_{1} E x(t)-z(t)-H_{2} D d(t)
$$

and the error dynamics is described by means of

$$
\dot{e}(t)=H_{1} E \dot{x}(t)-\dot{z}(t)-H_{2} D \dot{d}(t)
$$

Substituting (6) and (7) in (16) and by considering the convex property of scheduling functions (5) and the relations (8)-(11), the following equation is obtained:

$$
\begin{aligned}
\dot{e}(t) & =\sum_{i=1}^{h} \sum_{j=1}^{h} \rho_{i}(\theta(t)) \rho_{j}(\hat{\theta}(t))\left[\left(H_{1}\left(A_{j}+\Delta A_{i j}\right) x(t)\right.\right. \\
& +H_{1}\left(A_{j}^{d}+\Delta A_{i j}^{d}\right) x(t-\tau(t))-N_{j} z(t) \\
& -N_{j}^{d} z(t-\tau(t))-L_{j} y(t)-L_{j}^{d} y(t-\tau(t)) \\
& +H_{1}\left(B_{j}+\Delta B_{i j}\right) u(t)-G_{j} u(t)+H_{1} R_{i} d(t) \\
& \left.+H_{1}\left(F_{j}+\Delta F_{i j}\right) f(t)-W_{j} \hat{f}(t)-H_{2} D \dot{d}(t)\right]
\end{aligned}
$$

After some manipulations, (17) can be reformulated as follows:

$$
\begin{aligned}
\dot{e}(t) & =\sum_{i=1}^{h} \sum_{j=1}^{h} \rho_{i}(\theta(t)) \rho_{j}(\hat{\theta}(t))\left[N_{j} e(t)+N_{j}^{d} e(t-\tau(t))\right. \\
& +\left(H_{1} A_{j}-L_{j} C-N_{j} H_{1} E\right) x(t)+H_{1} \Delta A_{i j} x(t) \\
& +\left(H_{1} A_{j}^{d}-L_{j}^{d} C-N_{j}^{d} H_{1} E\right) x(t-\tau(t)) \\
& +H_{1} \Delta A_{i j}^{d} x(t-\tau(t))+\left(H_{1} B_{j}-G_{j}\right) u(t)+H_{1} \Delta B_{i j} u(t) \\
& +\left(H_{1} F_{j}-W_{j}\right) f(t)+W_{j}(f(t)-\hat{f}(t))+H_{1} \Delta F_{i j} f(t) \\
& +\left(H_{1} R_{i}+N_{j} H_{2} D-L_{j} D\right) d(t) \\
& \left.+\left(N_{j}^{d} H_{2} D-L_{j}^{d} D\right) d(t-\tau(t))-H_{2} D \dot{d}(t)\right] .
\end{aligned}
$$

If the following conditions are satisfied:

$$
\begin{gathered}
H_{1} E+H_{2} C=I_{n} \\
H_{1} A_{j}-L_{j} C-N_{j} H_{1} E=0
\end{gathered}
$$

$$
H_{1} A_{j}^{d}-L_{j}^{d} C-N_{j}^{d} H_{1} E=0
$$

$$
G_{j}=H_{1} B_{j}
$$

$$
W_{j}=H_{1} F_{j}
$$

then the observer error dynamics can be written as

$$
\begin{aligned}
\dot{e}(t) & =\sum_{i=1}^{h} \sum_{j=1}^{h} \rho_{i}(\theta(t)) \rho_{j}(\hat{\theta}(t))\left[N_{j} e(t)+H_{1} \Delta A_{i j} x(t)\right. \\
& +N_{j}^{d} e(t-\tau(t))+H_{1} \Delta A_{i j}^{d} x(t-\tau(t)) \\
& +H_{1} \Delta B_{i j} u(t)+W_{j} e_{f}(t)+H_{1} \Delta F_{i j} f(t) \\
& +\left(H_{1} R_{i}+N_{j} H_{2} D-L_{j} D\right) d(t) \\
& \left.+\left(N_{j}^{d} H_{2} D-L_{j}^{d} D\right) d(t-\tau(t))-H_{2} D \dot{d}(t)\right]
\end{aligned}
$$

where $e_{f}(t)$ is the fault estimation error defined as:

$$
e_{f}(t)=f(t)-\hat{f}(t)
$$

By introducing the following two variables:

$$
\begin{gathered}
K_{j}=L_{j}-N_{j} H_{2}, \\
K_{j}^{d}=L_{j}^{d}-N_{j}^{d} H_{2},
\end{gathered}
$$

the conditions (20)-(21), can be rewritten respectively as follows:

$$
\begin{aligned}
& N_{j}=H_{1} A_{j}-K_{j} C \\
& N_{j}^{d}=H_{1} A_{j}^{d}-K_{j}^{d} C
\end{aligned}
$$

and the error dynamics (24) is reduced to:

$$
\begin{aligned}
\dot{e}(t) & =\sum_{i=1}^{h} \sum_{j=1}^{h} \rho_{i}(\theta(t)) \rho_{j}(\hat{\theta}(t))\left[N_{j} e(t)+H_{1} \Delta A_{i j} x(t)\right. \\
& +N_{j}^{d} e(t-\tau(t))+H_{1} \Delta A_{i j}^{d} x(t-\tau(t))+H_{1} \Delta B_{i j} u(t) \\
& +W_{j} e_{f}(t)+H_{1} \Delta F_{i j} f(t)+\left(H_{1} R_{i}-K_{j} D\right) d(t) \\
& \left.-K_{j}^{d} D d(t-\tau(t))-H_{2} D \dot{d}(t)\right] .
\end{aligned}
$$


Assuming that the faults are constant or slow varying ( $\dot{f}(t) \cong 0$ ), according to (25) the fault estimation error dynamics is given by:

$$
\dot{e}_{f}(t)=-\dot{\hat{f}}(t)
$$

and according to (6), (31) is written as:

$$
\dot{e}_{f}(t)=-\sum_{j=1}^{h} \rho_{j}(\hat{\theta}(t))\left[\Lambda_{j} \operatorname{Ce}(t)+\Lambda_{j} D d(t)\right]
$$

In order to analyze the convergence of the state estimation error dynamics (30) and the fault estimation error dynamics (32), the following augmented system is constructed:

$$
\left\{\begin{array}{l}
\bar{E} \dot{\xi}(t)=\sum_{i=1}^{h} \sum_{j=1}^{h} \rho_{i}(\theta(t)) \rho_{j}(\hat{\theta}(t))\left[\bar{A}_{i j} \xi(t)\right. \\
\left.+\bar{A}_{i j}^{d} \xi(t-\tau(t))+\bar{R}_{i j} \bar{d}(t)\right] \\
\bar{e}(t)=\bar{C} \xi(t)
\end{array}\right.
$$

where the augmented state $\xi(t)$, augmented input $\bar{d}(t)$ and the output $\bar{e}(t)$ are respectively defined as:

$$
\begin{aligned}
& \xi(t)=\left[\begin{array}{ll:l}
e(t)^{T} & e_{f}(t)^{T} & x(t)^{T}
\end{array}\right]^{T}
\end{aligned}
$$

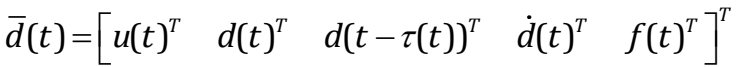

$$
\begin{aligned}
& \bar{e}(t)=\left[\begin{array}{ll}
e(t)^{T} & e_{f}(t)^{T}
\end{array}\right]^{T}
\end{aligned}
$$

and the matrices of system (33) are as follows:

$$
\begin{aligned}
& \bar{E}=\left[\begin{array}{cc:c}
I_{n} & 0 & 0 \\
0 & I_{k_{f}} & 0 \\
\hdashline 0 & 0 & E
\end{array}\right], \bar{A}_{i j}=\left[\begin{array}{cc:c}
N_{j} & W_{j} & H_{1} \Delta A_{i j} \\
-\Lambda_{j} C & 0 & 0 \\
\hdashline 0 & 0 & A_{i}
\end{array}\right], \\
& \bar{A}_{i j}^{d}=\left[\begin{array}{cc:c}
N_{j}^{d} & 0 & H_{1} \Delta A_{i j}^{d} \\
0 & 0 & 0 \\
\hdashline 0 & 0 & A_{i}^{d}
\end{array}\right], \bar{C}=\left[I_{n+k_{f}} \quad 0\right],
\end{aligned}
$$

$$
\bar{R}_{i j}=\left[\begin{array}{ccccc}
H_{1} \Delta B_{i j} & H_{1} R_{i}-K_{j} D & -K_{j}^{d} D & -H_{2} D & H_{1} \Delta F_{i j} \\
0 & -\Lambda_{j} D & 0 & 0 & 0 \\
\hdashline B_{i} & R_{i} & 0 & 0 & F_{i}
\end{array}\right] .
$$

Now, a lemma is introduced that will be used to guarantee the robust convergence of PI-UIO (6) in terms of robust stability of augmented error dynamics (33):

Lemma 1. The following SDLPV system is considered:

$$
\left\{\begin{aligned}
E \dot{x}(t) & =\sum_{i=1}^{h} \rho_{i}(\theta(t))\left[A_{i} x(t)+A_{i}^{d} x(t-\tau(t))\right. \\
& \left.+B_{i} w(t)\right] \\
z(t) & =C x(t)+D w(t)
\end{aligned}\right.
$$

in which $w(t)$ is a $L_{2}$-norm bounded exogenous input and $z(t)$ is the measured output and all the matrices are with compatible dimensions. $\tau_{m}$ and $\mu<1$ are the maximum values of the delay and its rate of change. For a given $\gamma>0$, if there exist matrices $P$ and $Q>0$ such that the following conditions hold for $i=1, \cdots, h$ :

$$
P^{T} E=E^{T} P \geq 0
$$

$$
\begin{gathered}
\Theta^{i}=\left[\begin{array}{cccc}
\Theta_{11}^{i} & P^{T} A_{i}^{d} & P^{T} B_{i} & C^{T} \\
* & -(1-\mu) e^{-2 \alpha \tau_{m}} Q & 0 & 0 \\
* & * & -\gamma^{2} I & D^{T} \\
* & * & * & -I
\end{array}\right]<0 \\
\Theta_{11}^{i}=P^{T} A_{i}+A_{i}^{T} P+2 \alpha P^{T} E+Q .
\end{gathered}
$$

then, system (37) is exponentially stable with the decay rate of $\alpha \geq 0$ for $w(t)=0$ and the attenuation condition $\|z(t)\|_{2}<\gamma\|w(t)\|_{2}$ holds for zero initial conditions.

Proof. The following Lyapunov-Krasovskii functional is considered:

$$
V\left(t, x_{t}\right)=x^{T}(t) P^{T} E x(t)+\int_{t-\tau(t)}^{t} x^{T}(\lambda) Q \mathrm{e}^{2 \alpha(\lambda-t)} x(\lambda) d \lambda
$$


in which $P^{T} E=E^{T} P \geq 0, Q=Q^{T}>0$ and $x_{t}:=x(\mathrm{t}+\omega)$ where $\omega \in\left[-\tau_{m}, 0\right]$. Consider the index:

$$
J=\int_{0}^{\infty}\left[z(t)^{T} z(t)-\gamma^{2} w(t)^{T} w(t)\right] d t
$$

For proving $\|z(t)\|_{2}<\gamma\|w(t)\|_{2}$ under zero initial conditions, it should be shown that $J<0$ holds in this case. The index $J$ is transformed as follows:

$$
\begin{aligned}
& J=\int_{0}^{\infty}\left[z(t)^{T} z(t)-\gamma^{2} w(t)^{T} w(t)+\dot{V}\left(t, x_{t}\right)+2 \alpha V\left(t, x_{t}\right)\right] d t \\
& -\int_{0}^{\infty} 2 \alpha V\left(t, x_{t}\right) d t+\left.V\left(t, x_{t}\right)\right|_{t=0}-\left.V\left(t, x_{t}\right)\right|_{t=\infty} .
\end{aligned}
$$

in which $\left.V\left(t, x_{t}\right)\right|_{t=0}=0$ and $\left.V\left(t, x_{t}\right)\right|_{t=\infty} \geq 0$ holds, thus:

$$
\begin{aligned}
& J \leq \int_{0}^{\infty}\left[z(t)^{T} z(t)-\gamma^{2} w(t)^{T} w(t)+\dot{V}(\right.\left(t, x_{t}\right) \\
&\left.+2 \alpha V\left(t, x_{t}\right)\right] d t .
\end{aligned}
$$

The time derivative of the Lyapunov-Krasovskii functional (40) is:

$$
\begin{aligned}
& \dot{V}\left(t, x_{t}\right)= \\
& \sum_{i=1}^{h} \rho_{i}(\theta(t))\left[x^{T}(t) P^{T} A_{i} x(t)+x^{T}(t) A_{i}^{T} P x(t)\right. \\
& +x^{T}(t) P^{T} A_{i}^{d} x(t-\tau(\mathrm{t}))+x^{T}(t-\tau(\mathrm{t}))\left(P^{T} A_{i}^{d}\right)^{T} x(t) \\
& \left.+x^{T}(t) P^{T} B_{i} w(t)+w^{T}(t)\left(P^{T} B_{i}\right)^{T} x(t)\right]+x^{T}(t) Q x(t) \\
& -(1-\dot{\tau}(\mathrm{t})) x^{T}(t-\tau(\mathrm{t})) e^{-2 \alpha \tau(\mathrm{t})} Q x(t-\tau(\mathrm{t})) \\
& -2 \alpha \int_{t-\tau(t)}^{t} x^{T}(\lambda) Q \mathrm{e}^{2 \alpha(\lambda-t)} x(\lambda) d \lambda
\end{aligned}
$$

Considering the convex property of the weighting functions (5) and the maximum bound of the delay derivative:

$$
J \leq \int_{0}^{\infty} \sum_{i=1}^{h} \rho_{i}(\theta(t)) \zeta(t)^{T} \Xi^{i} \zeta(t) d t
$$

where $\zeta(t)=\left[\begin{array}{lll}x(t)^{T} & x(t-\tau(t))^{T} \quad w(t)^{T}\end{array}\right]^{T}$ and

$$
\Xi^{i}=\left[\begin{array}{ccc}
\Xi_{11}^{i} & P^{T} A_{i}^{d} & P^{T} B_{i}+C^{T} D \\
* & -(1-\mu) e^{-2 \alpha \tau_{m}} Q & 0 \\
* & * & D^{T} D-\gamma^{2} I
\end{array}\right],
$$

$\Xi_{11}^{i}=P^{T} A_{i}+A_{i}^{T} P+2 \alpha P^{T} E+Q+C^{T} C$.

$\Xi^{i}<0$ assures $J<0$ such that by using the Schur Complement Lemma leads to (39), so the $H_{\infty}$ performance is guaranteed. The exponential stability of (37) is deduced from the negative definiteness of the following submatrix of $\Theta^{i}$ :

$$
\left[\begin{array}{cc}
P^{T} A_{i}+A_{i}^{T} P+2 \alpha P^{T} E+Q & P^{T} A_{i}^{d} \\
* & -(1-\mu) e^{-2 \alpha \tau_{m}} Q
\end{array}\right]
$$

which results in $\dot{V}\left(t, x_{t}\right)+2 \alpha V\left(t, x_{t}\right)<0$ in the non-actuated case. Therefore, the LMIs (38)-(39) are the sufficient condition for robust exponential stability of the system (37).

Remark 2. If system (37) satisfies $D=0$, then LMI (39) in Lemma 1, becomes

$$
\bar{\Theta}^{i}=\left[\begin{array}{ccc}
\bar{\Theta}_{11}^{i} & P^{T} A_{i}^{d} & P^{T} B_{i} \\
* & -(1-\mu) e^{-2 \alpha \tau_{m}} Q & 0 \\
* & * & -\gamma^{2} I
\end{array}\right]<0
$$

$\bar{\Theta}_{11}^{i}=P^{T} A_{i}+A_{i}^{T} P+2 \alpha P^{T} E+Q+C^{T} C$

by considering $D=0$ in (46).

\section{PI-UIO design and fault diagnosis}

\subsection{Description}

To obtain the PI-UIO matrices, (19) is reformulated in the following form:

$$
\underbrace{\left[\begin{array}{ll}
H_{1} & H_{2}
\end{array}\right]}_{\bar{H}}\left[\begin{array}{l}
E \\
C
\end{array}\right]=\underset{Y}{I_{n}}
$$

where $\bar{H} \in R^{n \times(n+m)}, Y \in R^{(n+m) \times n}$.

Remark 3. The matrix equation (49) is solvable if $\operatorname{rank}\left[\begin{array}{c}Y \\ \Psi\end{array}\right]=\operatorname{rank}(Y)$ which is equivalent to 


$$
\operatorname{rank}\left[\begin{array}{l}
E \\
C
\end{array}\right]=n
$$

Thus, under Assumption 3, the solution of (49) is:

$$
\bar{H}=\Psi Y^{+}+K\left(I_{n+m}-Y Y^{+}\right)
$$

where $Y^{+} \in R^{\left(n+h k_{d}\right) \times(n+m)}$ is the pseudo-inverse of $Y$. The term $K\left(I_{n+m}-Y Y^{+}\right)$adds an additional degree of freedom to the solution which helps to design a suitable PI-UIO. Equation (51) can be partitioned as:

$$
\begin{aligned}
\bar{H} & =\left[\begin{array}{ll}
H_{1} & H_{2}
\end{array}\right]=\Psi\left[\begin{array}{ll}
Y_{1}^{+} & Y_{2}^{+}
\end{array}\right]+K\left[\begin{array}{ll}
V_{1} & V_{2}
\end{array}\right] \\
= & {\left[\begin{array}{ll}
Y_{1}^{+}+K V_{1} & Y_{2}^{+}+K V_{2}
\end{array}\right] } \\
H_{10} & H_{20}
\end{aligned}
$$

in which $Y_{1}^{+}=Y^{+} T_{1}, Y_{2}^{+}=Y^{+} T_{2}, V_{1}=V T_{1}$ and $V_{2}=V T_{2}$ are calculated based on $T_{1}=\left[\begin{array}{ll}I_{n} & 0_{n \times m}\end{array}\right]^{T}, \quad T_{2}=\left[\begin{array}{ll}0_{m \times n} & I_{m}\end{array}\right]^{T}$ and $V=I_{n+m}-Y Y^{+}$.

\subsection{Main results}

Using the material introduced so far, the following theorem can be stated.

Theorem 1. Considering system (3), if there exist symmetric positive definite matrices $P_{1}, Q_{1}$ and $Q_{2}$, matrices $P_{2}, M, M_{j}$ and $M_{j}^{d}$ for $j=1, \ldots, h$ and positive scalar $\bar{\gamma}$ obtained as the solution to the following optimization problem:

$$
\min _{P_{1}, P_{2}, Q_{1}, Q_{2}, M, M_{j}, M_{j}^{d}} \bar{\gamma}
$$

subject to the following LMIs for $i=1, \ldots, h$ and $j=1, \ldots, h$ :

$$
\begin{gathered}
P_{2}^{T} E=E^{T} P_{2} \geq 0 \\
\Omega^{i j}=\left[\begin{array}{ccc}
\Omega_{11}^{i j} & \cdots & \Omega_{15}^{i j} \\
* & \ddots & \vdots \\
* & * & \Omega_{55}^{i j}
\end{array}\right]<0
\end{gathered}
$$

where

$$
\begin{aligned}
& \Omega_{11}^{i j}=\operatorname{sym}\left\{P_{1}\left[\begin{array}{cc}
H_{10} A_{j} & H_{10} F_{j} \\
0 & 0
\end{array}\right]+M\left[\begin{array}{ll}
V_{1} A_{j} & V_{1} F_{j}
\end{array}\right]\right. \\
& \left.-M_{j}\left[\begin{array}{ll}
C & 0
\end{array}\right]\right\}+2 \alpha P_{1}+Q_{1}+I_{n+k_{f}}
\end{aligned}
$$

$\Omega_{12}^{i j}=P_{1}\left[\begin{array}{c}H_{10} \Delta A_{i j} \\ 0\end{array}\right]+M V_{1} \Delta A_{i j}$

$\Omega_{13}^{i j}=P_{1}\left[\begin{array}{cc}H_{10} A_{j}^{d} & 0 \\ 0 & 0\end{array}\right]+M\left[V_{1} A_{j}^{d} \quad 0\right]-M_{j}^{d}\left[\begin{array}{ll}C & 0\end{array}\right]$

$\Omega_{14}^{i j}=P_{1}\left[\begin{array}{c}H_{10} \Delta A_{i j}^{d} \\ 0\end{array}\right]+M V_{1} \Delta A_{i j}^{d}$

$\Omega_{15}^{i j}=\left[P_{1}\left[\begin{array}{c}H_{10} \Delta B_{i j} \\ 0\end{array}\right]+M V_{1} \Delta B_{i j} \quad P_{1}\left[\begin{array}{c}H_{10} R_{i} \\ 0\end{array}\right]+M V_{1} R_{i}-M_{j} D \quad \ldots\right.$

$\left.\cdots \quad-M_{j}^{d} D-P_{1}\left[\begin{array}{c}H_{20} D \\ 0\end{array}\right]-M V_{2} D \quad P_{1}\left[\begin{array}{c}H_{10} \Delta F_{i j} \\ 0\end{array}\right]+M V_{1} \Delta F_{i j}\right]$

$\Omega_{22}^{i j}=\operatorname{sym}\left\{P_{2}^{T} A_{i}\right\}+2 \alpha P_{2}^{T} E+Q_{2}, \quad \Omega_{23}^{i j}=0$

$\Omega_{24}^{i j}=P_{2}^{T} A_{i}^{d}, \quad \Omega_{25}^{i j}=\left[\begin{array}{llllll}P_{2}^{T} B_{i} & P_{2}^{T} R_{i} & 0 & 0 & P_{2}^{T} F_{i}\end{array}\right]$

$\Omega_{33}^{i j}=-(1-\mu) e^{-2 \alpha \tau_{m}} Q_{1}, \quad \Omega_{34}^{i j}=0, \quad \Omega_{35}^{i j}=0$

$\Omega_{44}^{i j}=-(1-\mu) e^{-2 \alpha \tau_{m}} Q_{2}, \quad \Omega_{45}^{i j}=0, \quad \Omega_{55}^{i j}=-\bar{\gamma} I_{k_{u}+3 k_{d}+k_{f}}$

then, PI-UIO (6) with exponential decay rate $\alpha$ and the best achievable attenuation level $\gamma=\sqrt{\bar{\gamma}}$ for attenuating disturbance, noise and the uncertainty induced by inexact parameter measures exists. The matrices $K, K_{j}, \Lambda_{j}$ and $K_{j}^{d}$ for $j=1, \ldots, h$ are calculated with

$$
\begin{gathered}
K=\left(P_{1}\left[\begin{array}{c}
I_{n} \\
0_{k_{f} \times n}
\end{array}\right]\right)^{+} M \\
K_{j}=\left[\begin{array}{ll}
I_{n} & 0_{n \times k_{f}}
\end{array}\right]\left(P_{1}^{-1} M_{j}\right) \\
\Lambda_{j}=\left[\begin{array}{ll}
0_{k_{f} \times n} & I_{k_{f}}
\end{array}\right]\left(P_{1}^{-1} M_{j}\right)
\end{gathered}
$$




$$
K_{j}^{d}=\left(P_{1}\left[\begin{array}{c}
I_{n} \\
0_{k_{f} \times n}
\end{array}\right]\right)^{+} M_{j}^{d}
$$

Then, the matrices $H_{1}, H_{2}, N_{j}, N_{j}^{d}, L_{j}, L_{j}^{d}, G_{j}$ and $W_{j}$ are calculated based on (52), (52), (28), (29), (26), (27), (22) and (23), respectively.

Proof. In the matrices of the augmented system (33), based on the relations (23), (28), (29) and (52), the following reformulations are applied to the blocks corresponding to the dynamics of $\dot{e}(t)$ and $\dot{e}_{f}(t)$ :

$$
\begin{aligned}
& {\left[\begin{array}{cc}
N_{j} & W_{j} \\
-\Lambda_{j} C & 0
\end{array}\right]=\left[\begin{array}{cc}
H_{10} A_{j} & H_{10} F_{j} \\
0 & 0
\end{array}\right]+\left[\begin{array}{c}
K \\
0
\end{array}\right]\left[\begin{array}{ll}
V_{1} A_{j} & V_{1} F_{j}
\end{array}\right]} \\
& -\left[\begin{array}{c}
K_{j} \\
\Lambda_{j}
\end{array}\right]\left[\begin{array}{ll}
C & 0
\end{array}\right] \\
& {\left[\begin{array}{cc}
N_{j}^{d} & 0 \\
0 & 0
\end{array}\right]=\left[\begin{array}{cc}
H_{10} A_{j}^{d} & 0 \\
0 & 0
\end{array}\right]+\left[\begin{array}{c}
K \\
0
\end{array}\right]\left[\begin{array}{ll}
V_{1} A_{j}^{d} & 0
\end{array}\right]} \\
& -\left[\begin{array}{c}
K_{j}^{d} \\
0
\end{array}\right]\left[\begin{array}{ll}
C & 0
\end{array}\right] \\
& {\left[\begin{array}{c}
H_{1} \Delta A_{i j} \\
0
\end{array}\right]=\left[\begin{array}{c}
H_{10} \Delta A_{i j} \\
0
\end{array}\right]+\left[\begin{array}{c}
K \\
0
\end{array}\right] V_{1} \Delta A_{i j}} \\
& {\left[\begin{array}{c}
H_{1} \Delta A_{i j}^{d} \\
0
\end{array}\right]=\left[\begin{array}{c}
H_{10} \Delta A_{i j}^{d} \\
0
\end{array}\right]+\left[\begin{array}{c}
K \\
0
\end{array}\right] V_{1} \Delta A_{i j}^{d}} \\
& {\left[\begin{array}{ccccc}
H_{1} \Delta B_{i j} & H_{1} R_{i}-K_{j} D & -K_{j}^{d} D & -H_{2} D & H_{1} \Delta F_{i j} \\
0 & -\Lambda_{j} D & 0 & 0 & 0
\end{array}\right]=} \\
& {\left[\left[\begin{array}{c}
H_{10} \Delta B_{i j} \\
0
\end{array}\right]+\left[\begin{array}{c}
K \\
0
\end{array}\right] V_{1} \Delta B_{i j}\left[\begin{array}{c}
H_{10} R_{i} \\
0
\end{array}\right]+\left[\begin{array}{c}
K \\
0
\end{array}\right] V_{1} R_{i}-\left[\begin{array}{c}
K_{j} \\
\Lambda_{j}
\end{array}\right] D\right.} \\
& \left.-\left[\begin{array}{c}
K_{j}^{d} \\
0
\end{array}\right] D-\left[\begin{array}{c}
H_{20} D \\
0
\end{array}\right]-\left[\begin{array}{c}
K \\
0
\end{array}\right] V_{2} D \quad\left[\begin{array}{c}
H_{10} \Delta F_{i j} \\
0
\end{array}\right]+\left[\begin{array}{c}
K \\
0
\end{array}\right] V_{1} \Delta F_{i j}\right]
\end{aligned}
$$

Now, by considering the following block diagonal matrices for the Lyapunov-Krasovskii functional:

$$
P=\left[\begin{array}{cc}
P_{1} & 0 \\
0 & P_{2}
\end{array}\right]
$$

$$
Q=\left[\begin{array}{cc}
Q_{1} & 0 \\
0 & Q_{2}
\end{array}\right]
$$

where $P_{1}, Q_{1} \in R^{\left(n+k_{f}\right) \times\left(n+k_{f}\right)}$ and $P_{2}, Q_{2} \in R^{n \times n}$, Lemma 1 is applied to the augmented system (33). The condition (38) in this lemma is equivalent with $P_{2}^{T} E=E^{T} P_{2} \geq 0$ and $P_{1}=P_{1}^{T} \geq 0$ for system (33). By substituting the system state space matrices (33) in the LMIs (48) and using the formulation (60)(64), a set of nonlinear matrix inequalities are obtained due to multiplicative terms of some variables. By using the following change of variables:

$$
\begin{gathered}
M=P_{1}\left[\begin{array}{c}
K \\
0
\end{array}\right], \\
M_{j}=P_{1}\left[\begin{array}{l}
K_{j} \\
\Lambda_{j}
\end{array}\right], \\
M_{j}^{d}=P_{1}\left[\begin{array}{c}
K_{j}^{d} \\
0
\end{array}\right], \\
\bar{\gamma}=\gamma^{2}
\end{gathered}
$$

the nonlinearities are resolved and the set of LMIs (55) is obtained which assures the robust exponential convergence of the PI-UIO (6). When the optimization problem (53) under LMI conditions (54) and (55) is solved, then the unknown matrices of the observer can be calculated. By considering $P_{1}$ as $P_{1}=\left[\begin{array}{ll}P_{11} & P_{12}\end{array}\right],(67)$ is transformed to:

$$
M=P_{11} K=\left(P_{1}\left[\begin{array}{c}
I_{n} \\
0_{k_{f} \times n}
\end{array}\right]\right) K .
$$

So, $K$ is calculated based on (56). $K_{j}^{d}$ is calculated in a similar manner based on (59). According to (68):

$$
\left[\begin{array}{c}
K_{j} \\
\Lambda_{j}
\end{array}\right]=P_{1}^{-1} M_{j}
$$

Consequently, $K_{j}$ and $\Lambda_{j}$ are calculated from (57)-(58), respectively. With these variables, other unknown matrices of 
the observer can be calculated with the equations stated in the theorem the formulations of which have been derived in the previous sections.

Remark 4. Theorem 1 involves the non-strict condition $P_{2}^{T} E=E^{T} P_{2} \geq 0$ which contains equality constraint and may result in numerical problems. To avoid such problems, by parameterizing $P_{2}$ as $\mathcal{P}_{2}=P_{2} E+S X \quad$ where $\quad P_{2}>0 \quad$ and $X \in R^{(n-r) \times n}$ are the parameters and $S \in R^{n \times(n-r)}$ is any full column rank matrix which satisfies $E^{T} S=0$ [29], the following corollary can be proposed.

Corollary 1. Considering system (3), if there exist symmetric positive definite matrices $P_{1}, P_{2}, Q_{1}$ and $Q_{2}$, matrices $X, M$, $M_{j}$ and $M_{j}^{d}$ for $j=1, \ldots, h$ and positive scalar $\bar{\gamma}$ obtained as the solution to the following optimization problem:

$$
\min _{P_{1}, P_{2}, Q_{1}, Q_{2}, M, M_{j}, M_{j}^{d}, X} \bar{\gamma}
$$

subject to the following LMIs for $i=1, \ldots, h$ and $j=1, \ldots, h$ :

$$
\bar{\Omega}^{i j}=\left[\begin{array}{ccc}
\bar{\Omega}_{11}^{i j} & \cdots & \bar{\Omega}_{15}^{i j} \\
* & \ddots & \vdots \\
* & * & \bar{\Omega}_{55}^{i j}
\end{array}\right]<0
$$

where all blocks of $\bar{\Omega}^{i j}$ are identical with the corresponding blocks of $\Omega^{i j}$ defined in Theorem 1 , except the following blocks:

$$
\begin{aligned}
& \bar{\Omega}_{22}^{i j}=\operatorname{sym}\left\{\left(P_{2} E+S X\right)^{T} A_{i}\right\}+2 \alpha\left(P_{2} E+S X\right)^{T} E+Q_{2} \\
& \bar{\Omega}_{24}^{i j}=\left(P_{2} E+S X\right)^{T} A_{i}^{d} \\
& \bar{\Omega}_{25}^{i j}=\left[\begin{array}{ll}
\left(P_{2} E+S X\right)^{T} B_{i} & \left(P_{2} E+S X\right)^{T} R_{i}
\end{array}\right. \\
& \begin{array}{lll}
0 & 0 & \left.\left(P_{2} E+S X\right)^{T} F_{i}\right]
\end{array}
\end{aligned}
$$

in which $S \in R^{n \times(n-r)}$ is any full column rank matrix which satisfies $E^{T} S=0$. Then, PI-UIO (6) with exponential decay rate $\alpha$ and the best achievable attenuation level $\gamma=\sqrt{\bar{\gamma}}$ for attenuating disturbance, noise and the uncertainty induced by inexact parameter measures exists. The matrices $K, K_{j}, \Lambda_{j}$ and $K_{j}^{d}$ for $j=1, \ldots, h$ are calculated via (56)-(59) and the matrices $H_{1}, H_{2}, N_{j}, N_{j}^{d}, L_{j}, L_{j}^{d}, G_{j}$ and $W_{j}$ are calculated based on (52), (52), (28), (29), (26), (27), (22) and (23), respectively.

Remark 5. In system (1), the SDLPV system with a single delay is considered for the simplicity of notation. However, the results obtained could be extended to the case with multiple delays with the methodology being stated in [25].

Remark 6. This paper focuses on the SDLPV systems with inexact measured parameters. It is notable that the obtained results can also be used in the case of polytopic systems scheduling according to unmeasurable parameters such as $[9$, 17]. In this case, system (3) is scheduled according to $\rho_{i}(x(t))$ and PI-UIO (6) is scheduled according to $\rho_{j}(\hat{x}(t))$. Considering the substitution of $\rho_{i}(\theta(t))$ and $\rho_{j}(\hat{\theta}(t))$ respectively with $\rho_{i}(x(t))$ and $\rho_{j}(\hat{x}(t))$ in the relevant equations, the results obtained are also valid for the SDLPV systems with unmeasurable scheduling functions.

\subsection{Fault diagnosis}

The designed PI-UIO provides actuator fault estimates in addition to state estimates. Fault diagnosis is directly achieved via the fault estimates. In this direct fault diagnosis methodology, there is no need to first compute the residual signals and then evaluate them in order to detect the faults in the system. Fault isolation is also achieved directly with each of the fault estimated values. By eliminating the need of constructing a bank of observers and evaluating their residuals, the complexity of the design and computational burden of the fault diagnosis unit is reduced. Fault estimates 
and state estimates provided by this observer can be used to design the fault tolerant controller for SDLPV systems.

\subsection{Summary of the method}

The summary of the proposed method for designing PI-UIO for SDLPV systems in the case of inexact measured parameters is presented in Algorithm 1.

\section{Algorithm 1. PI-UIO design for SDLPV systems with inexact measured parameters}

Step 0. Check the conditions of Assumptions 2 and 3.

Step 1. Calculate $H_{10}=Y_{1}^{+}=Y^{+} T_{1}, H_{20}=Y_{2}^{+}=Y^{+} T_{2}, \quad V_{1}=V T_{1}$ and $V_{2}=V T_{2}$.

Step 2. Calculate $S \in R^{n \times(n-r)}$ with full column rank and satisfying $E^{T} S=0$.

Step 3. Solve the convex optimization problem (73) under LMI constraint (74) and obtain matrices $P_{1}, P_{2}, Q_{1}, Q_{2}, M, X$, $M_{j}$ and $M_{j}^{d}($ for $j=1, \ldots, h)$.

Step 4. Calculate $K, K_{j}, \Lambda_{j}$ and $K_{j}^{d}$ (for $\left.j=1, \ldots, h\right)$ from (56)-(59), respectively.

Step 5. Calculate $H_{1}$ and $H_{2}$ from (52).

Step 6. Calculate $N_{j}$ and $N_{j}^{d}$ (for $j=1, \ldots, h$ ) from (28) and (29), respectively.

Step 7. Calculate $L_{j}$ and $L_{j}^{d}$ (for $j=1, \ldots, h$ ) from (26) and (27), respectively.

Step 8. Calculate $G_{j}$ and $W_{j}$ (for $j=1, \ldots, h$ ) from (22) and (23), respectively.

\section{Illustrative example}

\subsection{Description}

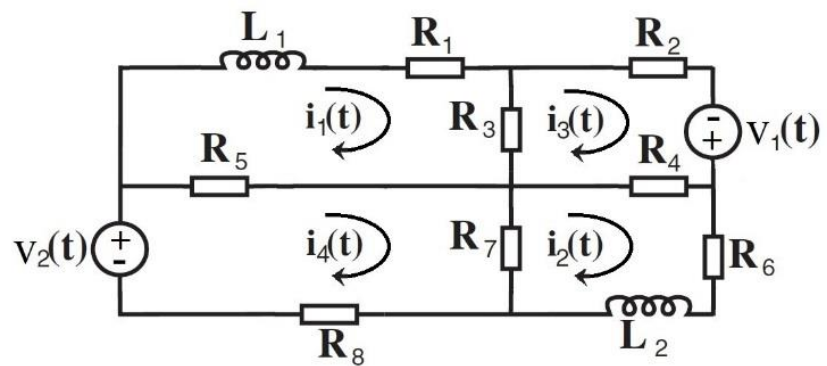

Fig. 1 An electrical circuit with four meshes

For illustrating the efficiency of the proposed method, an electrical circuit example with four meshes borrowed from [14] as shown in Fig. 1 is considered. In this circuit, there are eight resistors $R_{1}, \ldots, R_{8}$, two inductors $L_{1}$, $L_{2}$ and two voltage sources $V_{1}, V_{2}$. The values of the resistors and inductors are the same as [14] which are given in Table 1. Note that $R_{1}$ and $R_{6}$ are variable resistors for which the resistances vary according to the change of parameters $\theta_{1}(t)$ and $\theta_{2}(t)$, respectively. The range of variation of these two parameters are $\theta_{1}(t) \in[-0.5,0.5]$ and $\theta_{2}(t) \in[-1,1] . \quad i_{1}$ $, \ldots, i_{4}$ are the currents corresponding to the four meshes in the circuit.

The voltage sources have some delay to act since the input commands are sent through a communication network that presents some delays. This circuit is modeled using the Kirchhoff voltage law (KVL) leading to the following equations:

$\left\{\begin{array}{l}L_{1} \frac{d i_{1}}{d t}+R_{1} i_{1}+R_{3}\left(i_{1}-i_{3}\right)+R_{5}\left(i_{1}-i_{4}\right)=0 \\ L_{2} \frac{d i_{2}}{d t}+R_{7}\left(i_{2}-i_{4}\right)+R_{4}\left(i_{2}-i_{3}\right)+R_{6} i_{2}=0 \\ R_{2} i_{3}-v_{1}(t)+R_{4}\left(i_{3}-i_{2}\right)+R_{3}\left(i_{3}-i_{1}\right)=0 \\ R_{8} i_{4}-v_{2}(t)+R_{5}\left(i_{4}-i_{1}\right)+R_{7}\left(i_{4}-i_{2}\right)=0 \\ y_{1}(t)=v_{R_{5}}=R_{5}\left(i_{4}-i_{1}\right) \\ y_{2}(t)=v_{R_{7}}=R_{7}\left(i_{4}-i_{2}\right) \\ y_{1}(t)=v_{R_{4}}=R_{4}\left(i_{2}-i_{3}\right)\end{array}\right.$

The state vector is selected as $x(t)=\left[\begin{array}{llll}i_{1}(t) & i_{2}(t) & i_{3}(t) & i_{4}(t)\end{array}\right]^{T}$, the voltage source vector 
Table 1 - The values of circuit components

\begin{tabular}{ll}
\hline Component & Value \\
\hline$R_{1}$ & $\left(10+\theta_{1}(t)\right) \Omega$ \\
$R_{2}$ & $17 \Omega$ \\
$R_{3}$ & $3 \Omega$ \\
$R_{4}$ & $5 \Omega$ \\
$R_{5}$ & $2 \Omega$ \\
$R_{6}$ & $\left(27+\theta_{2}(t)\right) \Omega$ \\
$R_{7}$ & $8 \Omega$ \\
$R_{8}$ & $10 \Omega$ \\
$L_{1}$ & $0.3 H$ \\
$L_{2}$ & $0.65 H$
\end{tabular}

is $v(t)=\left[\begin{array}{ll}v_{1}(t) & v_{2}(t)\end{array}\right]^{T}$ which has a delay $\tau(t)$ associated to the input commands, so $v(t)=u(t-\tau(t))$. The delay value is $\tau(t)=0.6+0.4 \sin (t)$. The output vector is $y(t)=\left[\begin{array}{lll}y_{1}(t) & y_{2}(t) & y_{3}(t)\end{array}\right]^{T}$. The circuit's model is:

$$
\left\{\begin{array}{l}
E \dot{x}(t)=A(\theta(t)) x(t)+B u(t-\tau(t))+R d(t)+F f(t) \\
y(t)=C x(t)+D d(t)
\end{array}\right.
$$

where the matrices of the system are given by:

$$
\begin{gathered}
E=\left[\begin{array}{llll}
1 & 0 & 0 & 0 \\
0 & 1 & 0 & 0 \\
0 & 0 & 0 & 0 \\
0 & 0 & 0 & 0
\end{array}\right], B=F=\left[\begin{array}{ll}
0 & 0 \\
0 & 0 \\
1 & 0 \\
0 & 1
\end{array}\right], R=\left[\begin{array}{c}
0 \\
0 \\
0.7 \\
0.2
\end{array}\right], \\
A(\theta(t))=\left[\begin{array}{cccc}
-\frac{R_{11}+\theta_{1}(t)}{L_{1}} & 0 & \frac{R_{3}}{L_{1}} & \frac{R_{5}}{L_{1}} \\
0 & -\frac{R_{22}+\theta_{2}(t)}{L_{2}} & \frac{R_{4}}{L_{2}} & \frac{R_{7}}{L_{2}} \\
R_{3} & R_{4} & -R_{33} & 0 \\
R_{5} & R_{7} & 0 & -R_{44}
\end{array}\right], \\
C=\left[\begin{array}{cccc}
-R_{5} & 0 & 0 & R_{5} \\
0 & -R_{7} & 0 & R_{7} \\
0 & R_{4} & -R_{4} & 0
\end{array}\right], D=\left[\begin{array}{c}
0.2 \\
0.1 \\
0.5
\end{array}\right],
\end{gathered}
$$

where

$$
\begin{aligned}
& R_{11}=\bar{R}_{1}+R_{3}+R_{5}, R_{22}=R_{4}+\bar{R}_{6}+R_{7}, \\
& R_{33}=R_{2}+R_{3}+R_{4}, R_{44}=R_{5}+R_{7}+R_{8} .
\end{aligned}
$$

The model of the circuit is a singular LPV system with input delay. To use the approach presented in this paper for state delayed singular LPV systems, the two inputs are considered as additional states. By choosing $\widehat{x}(t)=\left[\begin{array}{llllll}i_{1}(t) & i_{2}(t) & i_{3}(t) & i_{4}(t) & u_{1}(t) & u_{2}(t)\end{array}\right]^{T} \quad$ and $\hat{y}(t)=\left[\begin{array}{lllll}y_{1}(t) & y_{2}(t) & y_{3}(t) & u_{1}(t) & u_{2}(t)\end{array}\right]^{T}, \quad$ the following SDLPV system is obtained:

$$
\left\{\begin{array}{c}
\hat{E} \dot{x}(t)=\hat{A}(\theta(t)) \widehat{x}(t)+\widehat{A}^{d} \widehat{x}(t-\tau(t))+\widehat{B} u(t) \\
\quad+\widehat{R} d(t)+\widehat{F} f(t) \\
\widehat{y}(t)=\widehat{C} \hat{x}(t)+\widehat{D} d(t)
\end{array}\right.
$$

where

$$
\begin{gathered}
\widehat{E}=\left[\begin{array}{ll}
E & 0 \\
0 & 0
\end{array}\right], \widehat{A}(\theta(t))=\left[\begin{array}{cc}
A(\theta(t)) & 0 \\
0 & -I_{k_{u}}
\end{array}\right], \widehat{A}^{d}=\left[\begin{array}{ll}
0 & B \\
0 & 0
\end{array}\right], \\
\widehat{B}=\left[\begin{array}{c}
0 \\
I_{k_{u}}
\end{array}\right], \widehat{R}=\left[\begin{array}{c}
R \\
0
\end{array}\right], \widehat{F}=\left[\begin{array}{c}
F \\
0
\end{array}\right], \widehat{C}=\left[\begin{array}{cc}
C & 0 \\
0 & I_{k_{u}}
\end{array}\right], \widehat{D}=\left[\begin{array}{l}
D \\
0
\end{array}\right] .
\end{gathered}
$$

System (77) is a SDLPV system with state delay in the form of (1). This system can be converted to polytopic representation (3) with the methodology recalled in Remark 1 . There are two parameters in the system (77), thus there are four subsystems in the polytopic representation. The gain matrices of these subsystems are calculated as follows:

$$
\begin{aligned}
& \widehat{A}_{1}=\left.\hat{A}(\theta(t))\right|_{\substack{\theta_{1}(t)=\theta_{1}^{m} \\
\theta_{2}(t)=\theta_{2}^{m}}}, \hat{A}_{2}=\left.\hat{A}(\theta(t))\right|_{\substack{\theta_{1}(t)=\theta_{1}^{M} \\
\theta_{2}(t)=\theta_{2}^{m}}}, \\
& \widehat{A}_{3}=\left.\hat{A}(\theta(t))\right|_{\substack{\theta_{1}(t)=\theta_{1}^{m} \\
\theta_{2}(t)=\theta_{2}^{m}}}, \hat{A}_{4}=\left.\hat{A}(\theta(t))\right|_{\substack{\theta_{1}(t)=\theta_{1}^{M} \\
\theta_{2}(t)=\theta_{2}^{n}}}
\end{aligned}
$$

where $\hat{A}_{i}$ for $i=1, \cdots, 4$ is the corresponding matrix in the subsystem $i$ and the other matrices are constant as defined in (77). The corresponding time varying weights of the four subsystems in representation (3) are calculated as follows:

$$
\begin{aligned}
& \rho_{1}(\theta(t))=\alpha_{1}(t) \alpha_{2}(t), \rho_{4}(\theta(t))=\left(1-\alpha_{1}(t)\right)\left(1-\alpha_{2}(t)\right) \\
& \rho_{2}(\theta(t))=\left(1-\alpha_{1}(t)\right) \alpha_{2}(t), \rho_{3}(\theta(t))=\alpha_{1}(t)\left(1-\alpha_{2}(t)\right)
\end{aligned}
$$

where $\alpha_{1}(t)=\frac{\theta_{1}^{M}-\theta_{1}(t)}{\theta_{1}^{M}-\theta_{1}^{m}}$ and $\alpha_{2}(t)=\frac{\theta_{2}^{M}-\theta_{2}(t)}{\theta_{2}^{M}-\theta_{2}^{m}}$. 


\subsection{Results}

Now, a PI-UIO is designed for the system according to Algorithm 1. The convex optimization (73) is solved with the SeDumi solver [30] via the YALMIP toolbox [31]. The parameter $\alpha$ is set to 0 . The PI-UIO matrices are calculated based on the steps of Algorithm 1. Although there are four subsystems, only the results for the first subsystem are presented here due to space limitation:

$$
\begin{gathered}
N_{1}=\left[\begin{array}{rrrr}
38.6072 & 135.6684 & -89.4444 & \\
-148.7445 & -178.2624 & 164.4025 & \\
63.6216 & 171.8490 & -129.9844 & \\
-165.2371 & -215.7886 & 211.3339 & \\
-0.0000 & -0.0000 & 0.0000 & \\
-0.0000 & 0.0000 & 0.0000 & \\
& -77.1500 & -0.0000 & -0.0000 \\
58.5234 & -0.0000 & -0.0000 \\
-109.4459 & -0.0000 & -0.0000 \\
& 115.5447 & 0.0000 & -0.0000 \\
& -0.0000 & -1.5179 & -0.0000 \\
-0.0000 & -0.0000 & -1.5179
\end{array}\right] \\
N_{1}^{d}=10^{-5} \times\left[\begin{array}{ccccc}
-0.0600 & 0.5673 & -0.0094 & \\
0.0037 & 0.7598 & -0.0874 & \\
0.0491 & 0.6150 & -0.1118 & \\
-0.0565 & 0.7623 & -0.0342 & \\
0.0723 & -0.0181 & -0.0622 & \\
0.0302 & 0.1079 & -0.0388 & \\
& -0.4978 & 0.6071 & 0.4274 \\
& -0.6761 & 0.8031 & 0.6186 \\
& -0.5524 & 0.5325 & 0.3155 \\
& -0.6717 & 0.8111 & 0.7277 \\
& 0.0081 & 0.0111 & 0.0586 \\
& -0.0993 & -0.0038 & -0.0764
\end{array}\right]
\end{gathered}
$$

$$
\begin{aligned}
L_{1} & =\left[\begin{array}{rrrrr}
4.6566 & 1.9341 & -10.8717 & 0.0000 & 0.0000 \\
7.6451 & 2.1753 & 11.7865 & -0.0000 & -0.0000 \\
-1.6557 & 0.6966 & -10.3958 & -0.0000 & 0.0000 \\
22.5861 & 0.1105 & 7.5244 & 0.0000 & -0.0000 \\
-0.0000 & 0.0000 & 0.0000 & -0.0000 & -0.0000 \\
0.0000 & -0.0000 & 0.0000 & -0.0000 & -0.0000
\end{array}\right] \\
L_{1}^{d} & =\left[\begin{array}{ccccc}
0.0000 & -0.0000 & 0.0000 & -1.7732 & -0.9096 \\
0.0000 & -0.0000 & 0.0000 & 2.6749 & -1.0496 \\
0.0000 & -0.0000 & 0.0000 & -1.6660 & 0.1801 \\
0.0000 & -0.0000 & 0.0000 & 1.8106 & -1.6813 \\
0.0000 & -0.0000 & 0.0000 & 0.0000 & 0.0000 \\
-0.0000 & 0.0000 & 0.0000 & 0.0000 & 0.0000
\end{array}\right] \\
\Lambda_{1} & =\left[\begin{array}{ccccc}
-0.1036 & -1.6934 & 0.7416 & -0.0000 & -0.0000 \\
11.3989 & -0.5570 & -7.2003 & -0.0000 & -0.0000
\end{array}\right]
\end{aligned}
$$

The other PI-UIO matrices are constant and their values are:

$$
\begin{gathered}
G=10^{-11} \times\left[\begin{array}{rr}
-0.0000 & -0.0000 \\
0.0000 & 0.0000 \\
0.0000 & -0.0000 \\
-0.0000 & 0.0000 \\
0.1599 & 0.0000 \\
0.0000 & 0.1363
\end{array}\right], W=\left[\begin{array}{rrr}
-1.7732 & -0.9096 \\
2.6749 & -1.0496 \\
-1.6660 & 0.1801 \\
1.8106 & -1.6813 \\
0.0000 & 0.0000 \\
0.0000 & 0.0000
\end{array}\right] \\
H_{2}=\left[\begin{array}{ccccc}
0.0065 & -0.0016 & -0.0000 & 0 & 0 \\
0.0538 & -0.0135 & 0.0000 & 0 & 0 \\
0.5711 & -0.1428 & -0.2000 & 0 & 0 \\
-0.0108 & 0.1277 & 0.0000 & 0 & 0 \\
-0.0000 & 0.0000 & 0.0000 & 1.0000 & 0 \\
-0.0000 & 0.0000 & 0.0000 & 0 & 1.0000
\end{array}\right]
\end{gathered}
$$

\subsection{Simulation}

System (77) with the PI-UIO that was designed in the previous section has been simulated. $u_{1}(t)=5+\cos (3 t)$ and $u_{2}(t)=\sin (2 t)$ are applied as the system inputs. The parameters' variation are $\theta_{1}(t)=0.5 \sin (0.3 t)$ and $\theta_{2}(t)=\cos (0.5 t)$. The parameter measures are corrupted with two zero-mean noises with standard deviations equal to 0.2 and 0.3 , respectively. The disturbance input is a zero mean noise with standard deviation of 0.2 . Different scenarios are used for testing the proposed approach in the considered case 

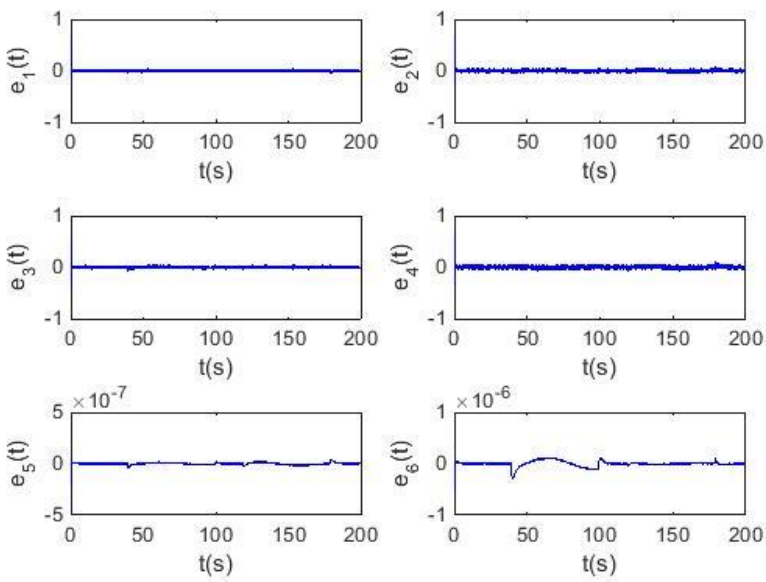

Fig. 2 State estimation errors in the first scenario
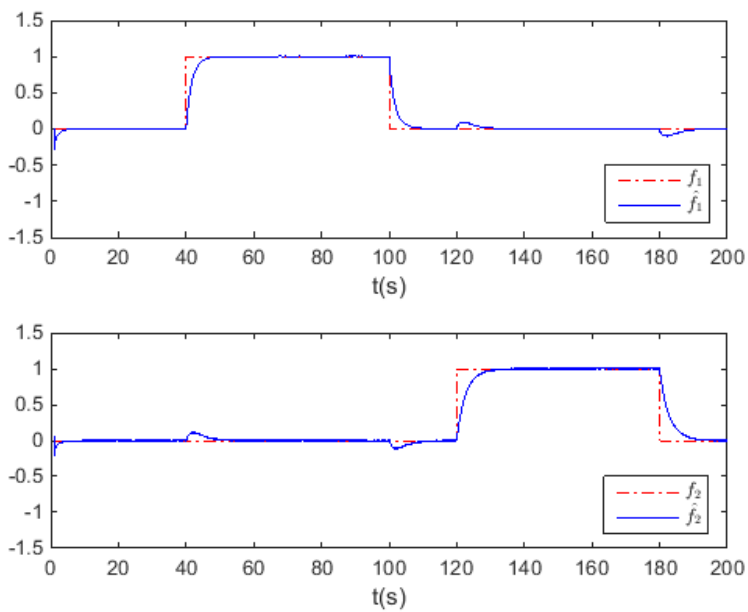

Fig. 3 Abrupt faults and their estimates

study. In the first scenario, abrupt faults occur on the two sources in the time intervals $[40,100]$ and $[120,180]$ seconds, respectively. The state estimation errors are shown in Fig. 2. It can be seen that the estimation error is robustly bounded in spite of input and output noises and uncertainty induced by inexact parameter measures. The fault estimation results are depicted in Fig. 3. As it can be observed from this figure, the constant faults on the actuators are detected and estimated without steady state error. The abrupt change that occurs in one fault causes a small distortion on the other fault estimate. This distortion occurs because when sudden faults happen in the system, the assumption of slow varying faults $(\dot{f}(t) \cong 0)$ is not satisfied. At such moments, the derivative of the fault is very large and the coupling which exists in the system dynamics causes such a distortion. Some approaches have been proposed in the literature for time varying faults such as the PMI observer [9] and adaptive observer [14]. However, these approaches cannot eliminate the distortion caused by abrupt changes in the fault because they assume that the fault derivative is bounded which is not the case when faults with sudden changes are considered.

The second scenario considers the case of incipient faults occurring in the system. The faults on the first and second actuators start to grow in $t=40 \mathrm{~s}$ and $t=120 \mathrm{~s}$, respectively. The faults and their estimates are shown in Fig. 4. It can be observed that the incipient faults are also detected and isolated directly based on their estimated values as they start to grow in the system. Early diagnosis of incipient faults helps to activate the fault tolerant action before it leads to any serious damage.

In the third scenario, time varying sinusoidal faults with frequency of $\omega=0.1 \mathrm{rad} / \mathrm{s}$ occur on the two actuators. The result of fault estimation is plotted in Fig. 5. The PI-UIO has steady state error in estimating time varying faults because it is designed for constant and slow varying faults. If the frequency of the sinusoidal fault increases, the steady state error will increase because the assumption $\dot{f}(t) \cong 0$ is not
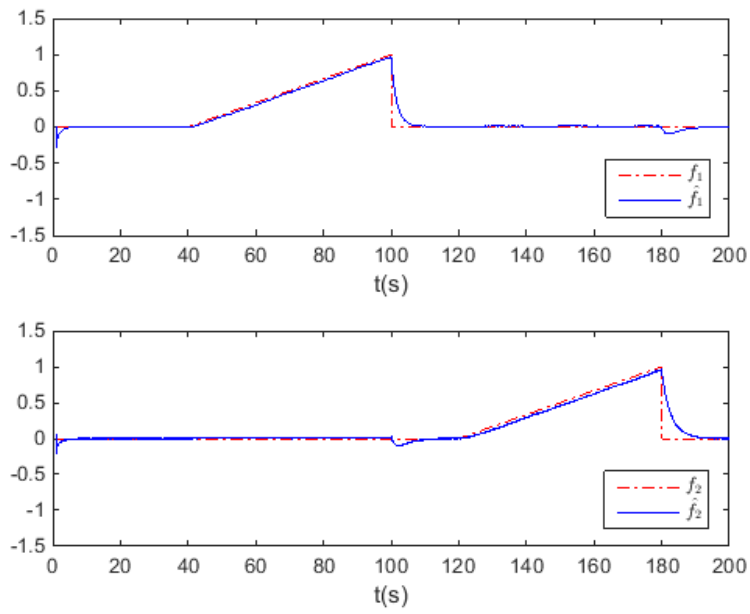

Fig. 4 Incipient faults and their estimates 

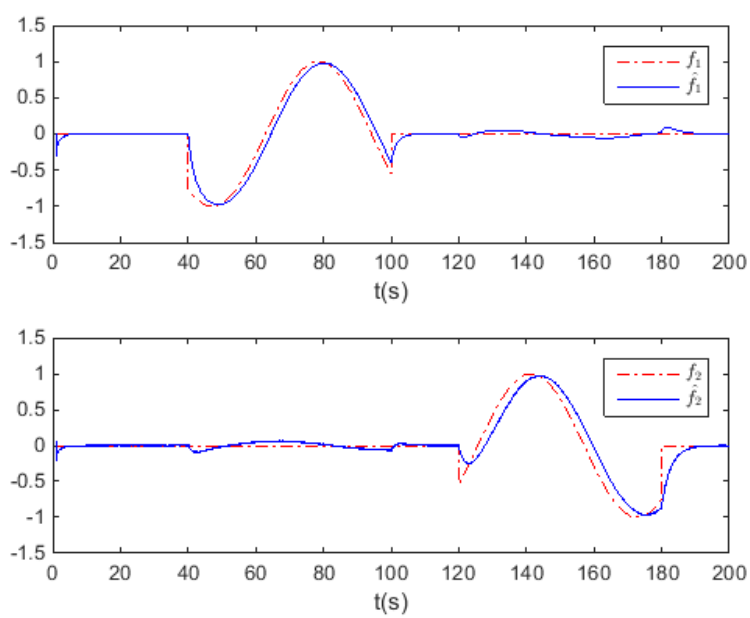

Fig. 5 Time varying faults and their estimates

satisfied. Relaxing this assumption is part of the future research which can be developed considering the use of PMI or adaptive observer following the references provided in the introduction. Reducing the estimation error of time varying faults will improve the performance of the active fault tolerant controller.

\section{Conclusion}

In this paper, a polytopic PI-UIO was designed for SDLPV systems. In the considered system, there are delayed dynamics, input disturbance, output noise and actuator faults. The case of inexact measured parameters was considered closer to the real situations. The system with uncertainty induced by inexact measured parameters was formulated as an uncertain system. The proposed observer can carry out both state and actuator fault estimation. Fault diagnosis is directly achieved based on the estimated values of the faults which avoids the use of the residual computation and evaluation, reducing the computational burden of the diagnosis unit. The effects of input disturbance, output noise and uncertainty of inexact measured parameters are robustly attenuated on the state and fault estimation error. The robust exponential stability of the error dynamics of the observer and the calculation of the observer matrices were formulated as a convex optimization problem with LMI constraints. An electrical circuit was used as a case study to show the efficiency of the proposed state and fault estimation method. Extending the results for the case of fast time varying faults is part of the future research.

\section{REFERENCES}

1. Zarei, J. and Shokri, E., 'Robust Sensor Fault Detection Based on Nonlinear Unknown Input Observer', Measurement, 2014, 48, pp. 355-367.

2. Lee, K.-S. and Park, T.-G., 'New Results on Fault Reconstruction Using a Finite-Time Converging Unknown Input Observer', IET Control Theory \& Applications, 2012, 6, (9), pp. 1258-1265.

3. Ahmadizadeh, S., Zarei, J., and Karimi, H.R., 'Robust Unknown Input Observer Design for Linear Uncertain Time Delay Systems with Application to Fault Detection', Asian Journal of Control, 2014, 16, (4), pp. 1006-1019.

4. Rotondo, D., Witczak, M., Puig, V., Nejjari, F., and Pazera, M., 'Robust Unknown Input Observer for State and Fault Estimation in Discrete-Time Takagi-Sugeno Systems', International Journal of Systems Science, 2016, 47, (14), pp. 3409-3424.

5. de Oca, S., Puig, V., Witczak, M., and Dziekan, Ł., 'FaultTolerant Control Strategy for Actuator Faults Using Lpv Techniques: Application to a Two Degree of Freedom Helicopter', International Journal of Applied Mathematics and Computer Science, 2012, 22, (1), pp. 161-171.

6. Aguilera-González, A., Theilliol, D., Adam-Medina, M., Astorga-Zaragoza, C., and Rodrigues, M., 'Sensor Fault and Unknown Input Estimation Based on Proportional Integral Observer Applied to Lpv Descriptor Systems', IFAC Proceedings Volumes, 2012, 45, (20), pp. 10591064.

7. Hamdi, H., Rodrigues, M., Mechmeche, C., Theilliol, D., and Braiek, N.B., 'Fault Detection and Isolation in Linear Parameter-Varying Descriptor Systems Via Proportional Integral Observer', International journal of adaptive control and signal processing, 2012, 26, (3), pp. 224-240.

8. Li, X. and Zhu, F., 'Simultaneous Time-Varying Actuator and Sensor Fault Reconstruction Based on Pi Observer for Lpv Systems', International journal of adaptive control and signal processing, 2015, 29, (9), pp. 1086-1098.

9. Ichalal, D., Marx, B., Ragot, J., and Maquin, D., 'Simultaneous State and Unknown Inputs Estimation with $\mathrm{Pi}$ and Pmi Observers for Takagi Sugeno Model with Unmeasurable Premise Variables', in, Control and Automation, 2009. MED'09. 17th Mediterranean Conference on, (IEEE, 2009)

10. Duan, G.-R., Analysis and Design of Descriptor Linear Systems, (Springer Science \& Business Media, 2010)

11. Zhang, Q., Liu, C., and Zhang, X., Complexity, Analysis and Control of Singular Biological Systems, (Springer Science \& Business Media, 2012) 
12. Wang, Z., Rodrigues, M., Theilliol, D., and Shen, Y., 'Fault Estimation Filter Design for Discrete-Time Descriptor Systems', IET Control Theory \& Applications, 2015, 9, (10), pp. 1587-1594.

13. Yao, L., Cocquempot, V., and Wang, H., 'Fault Diagnosis and Fault Tolerant Control Scheme for a Class of NonLinear Singular Systems', IET Control Theory \& Applications, 2014, 9, (6), pp. 843-851.

14. Rodrigues, M., Hamdi, H., Theilliol, D., Mechmeche, C., and BenHadj Braiek, N., 'Actuator Fault Estimation Based Adaptive Polytopic Observer for a Class of Lpv Descriptor Systems', International Journal of Robust and Nonlinear Control, 2015, 25, (5), pp. 673-688.

15. López-Estrada, F.-R., Ponsart, J.-C., Astorga-Zaragoza, C.-M., Camas-Anzueto, J.-L., and Theilliol, D., 'Robust Sensor Fault Estimation for Descriptor-Lpv Systems with Unmeasurable Gain Scheduling Functions: Application to an Anaerobic Bioreactor', International Journal of Applied Mathematics and Computer Science, 2015, 25, (2), pp. 233-244.

16. Jia, Q., Chen, W., Zhang, Y., and Chen, X., 'Robust Fault Reconstruction Via Learning Observers in Linear Parameter-Varying Systems Subject to Loss of Actuator Effectiveness', IET Control Theory \& Applications, 2014, 8, (1), pp. 42-50.

17. Estrada, F.L., Ponsart, J., Theilliol, D., and AstorgaZaragoza, C., 'Robust H-/Hळ Fault Detection Observer Design for Descriptor-Lpv Systems with Unmeasurable Gain Scheduling Functions', International Journal of Control, 2015, 88, (11), pp. 2380-2391.

18. Boulaabi, I., Sellami, A., and Hmida, F.B., 'Robust DelayDerivative-Dependent Sliding Mode Observer for Fault Reconstruction: A Diesel Engine System Application', Circuits, Systems, and Signal Processing, 2015, pp. 1-22.

19. Zhao, D., Shen, D., and Wang, Y., 'Fault Diagnosis and Compensation for Two-Dimensional Discrete Time Systems with Sensor Faults and Time-Varying Delays', International Journal of Robust and Nonlinear Control, 2017.

20. Jiang, B., Zhang, K., and Shi, P., 'Less Conservative Criteria for Fault Accommodation of Time-Varying Delay Systems Using Adaptive Fault Diagnosis Observer', International journal of adaptive control and signal processing, 2010, 24, (4), pp. 322-334.
21. Sadaka, H., Shafai, B., and Sipahi, R., 'Design of Pi Observer for Disturbance Attenuation and Fault Detection in Linear Time-Delay Systems', in, Asian Control Conference, 2009. ASCC 2009. 7th, (IEEE, 2009)

22. Li, F. and Zhang, X., 'Delay-Range-Dependent Robust $\mathrm{H} \infty$ Filtering for Singular Lpv Systems with Time Variant Delay', International Journal of Innovative Computing, Information and Control, 2013, 9, (1), pp. 339-353.

23. Li, F. and Zhang, X., 'A Delay-Dependent Bounded Real Lemma for Singular Lpv Systems with Time-Variant Delay', International Journal of Robust and Nonlinear Control, 2012, 22, (5), pp. 559-574.

24. Zhang, X. and Zhu, H., 'Robust Stability and Stabilization Criteria for Discrete Singular Time-Delay Lpv Systems', Asian Journal of Control, 2012, 14, (4), pp. 1084-1094.

25. Hassanabadi, A.H., Shafiee, M., and Puig, V., 'Uio Design for Singular Delayed Lpv Systems with Application to Actuator Fault Detection and Isolation', International Journal of Systems Science, 2016a, 47, (1), pp. 107-121.

26. Hassanabadi, A.H., Shafiee, M., and Puig, V., State and Fault Estimation of Singular Delayed Lpv Systems Via Proportional-Integral Observer', the 3rd Conference on Control and Fault-Tolerant Systems, (2016)

27. Hassanabadi, A.H., Shafiee, M., and Puig, V., 'Robust Fault Detection of Singular Lpv Systems with Multiple Time-Varying Delays', International Journal of Applied Mathematics and Computer Science, 2016, 26, (1), pp. 45-61.

28. Ichalal, D., Marx, B., Ragot, J., and Maquin, D., 'Fault Diagnosis in Takagi-Sugeno Nonlinear Systems.', in, 7th IFAC Symposium on Fault Detection, Supervision and Safety of Technical Processes, Safeprocess, (2009)

29. Lam, J. and $\mathrm{Xu}$, S., Robust Control and Filtering of Singular Systems, (Springer-Verlag Berlin/Heidelberg, 2006)

30. Sturm, J.F., 'Using Sedumi 1.02, a Matlab Toolbox for Optimization over Symmetric Cones', Optimization methods and software, 1999, 11, (1-4), pp. 625-653.

31. Löfberg, J., 'Yalmip: A Toolbox for Modeling and Optimization in Matlab.', in, IEEE International Symposium on Computer Aided Control Systems Design, (IEEE, 2004) 